\title{
Surgery of Tuberculum Sellae Meningioma: A Technical Purview on Pterional Approach
}

\author{
Anil Nanda ${ }^{1}$ Devi Prasad Patra ${ }^{1}$ Amey Rajan Savardekar ${ }^{1} \quad$ Nasser Mohammed $^{1}$ Vinayak Narayan ${ }^{1}$ \\ Shyamal C. Bir ${ }^{1}$ \\ ${ }^{1}$ Department of Neurosurgery, Louisiana State University Health \\ Sciences Centre, Shreveport, Louisiana, United States \\ J Neurol Surg B 2018;79(suppl S3):S265-S266. \\ Address for correspondence Anil Nanda, MD, FACS, Department of \\ Neurosurgery, Louisiana State University Health-Shreveport, 1501 \\ Kings Highway, Shreveport, LA 71130-3932, United States \\ (e-mail: ananda@lsuhsc.edu).
}

\begin{abstract}
Keywords

- tuberculum sellae meningioma

- surgery

- operative video

- transcranial approach

- technical nuances

Objective Amidst the raging debate between transnasal and transcranial approaches, the critical factor that dictates success lies in the meticulous dissection of the tumor from the optic apparatus, anterior communicating artery complex, and the pituitary stalk. In this surgical video, we describe the resection of a tuberculum sellae meningioma through the pterional approach highlighting dissection through the appropriate arachnoidal plane.

Case Description The patient is a 75-year-old female who presented with optic nerve compression with bitemporal hemianopia and endocrine function abnormalities. A transcranial approach was done to effectively decompress the optic nerve. The video includes a detailed discussion on the surgical techniques including working between the different surgical corridors, working in the arachnoid plane to dissect the neurovascular structures away from the tumor, identification, and preservation of pituitary stalk. Postoperatively, the patient's visual field deficit improved with a remaining isolated right temporal field defect at 2 months follow-up. Her endocrine function improved after the surgery.

Conclusion In the era of endoscopic surgery, transcranial approaches for suprasellar tumors like tuberculum sellae meningiomas still have a distinct surgical role. Our discussion extends to reiterate the strengths and weaknesses of the transcranial approach to this region as compared with the endoscopic approach. We believe decompression of optic nerves, separation of Acom complex from the tumor, and preservation of pituitary stalk are better achieved through a transcranial approach. The link to the video can be found at: https://youtu.be/kw5eP3oUd6Q.
\end{abstract}

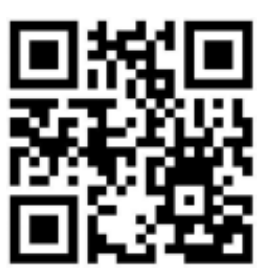

received

October 16, 2017

accepted

December 14, 2017

published online

February 16, 2018 www.thieme.com/skullbasevideos

www.thieme.com/jnlsbvideos
DOI https://doi.org/

10.1055/s-0038-1625945. ISSN 2193-6331. (c) 2018 Georg Thieme Verlag KG Stuttgart · New York
License terms

(c) (i) $\ominus$ (\$) 
S266 Surgery of Tuberculum Sellae Meningioma: A Technical Purview on Pterional Approach Nanda et al.

Conflict of Interest

None.

Funding

None.

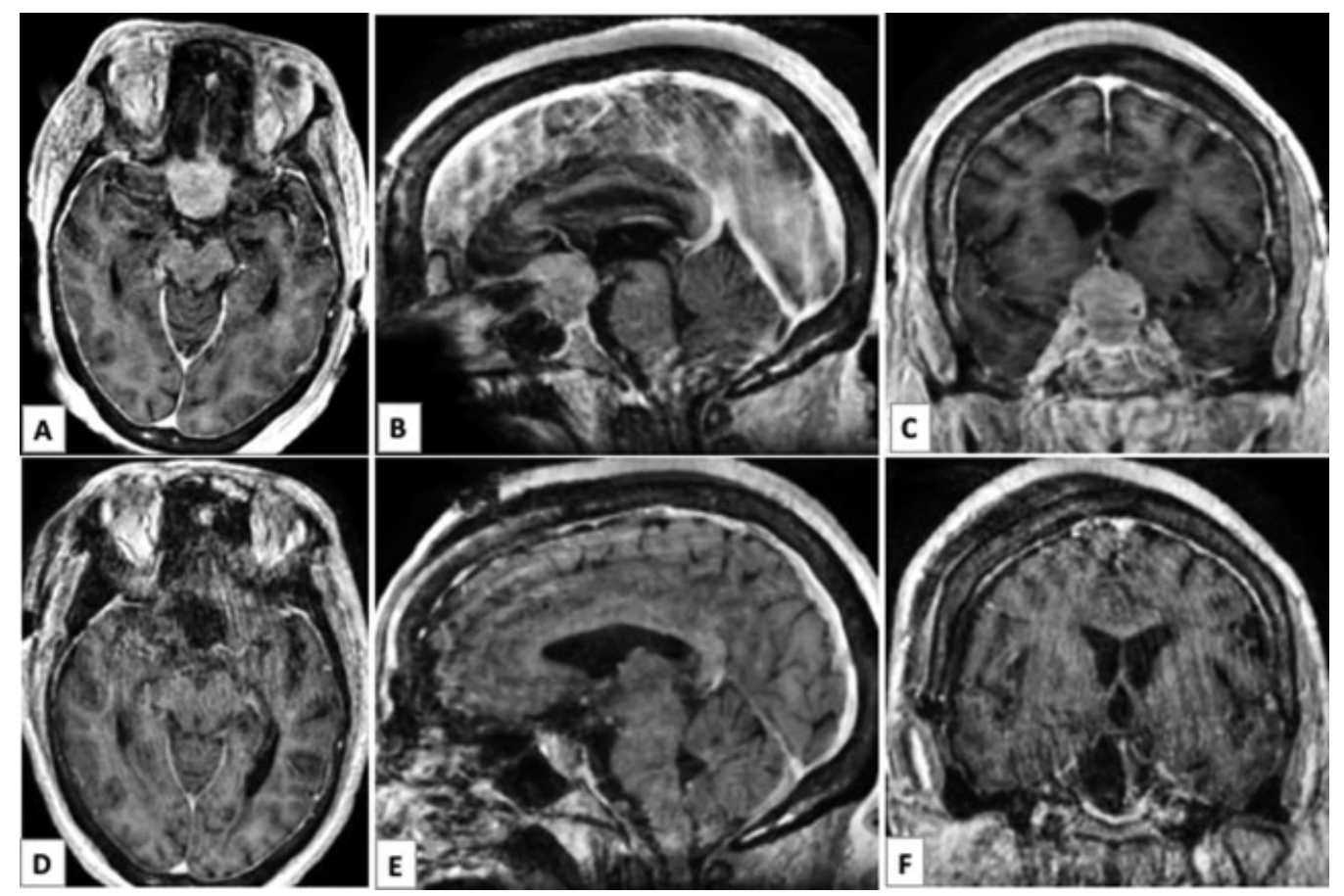

Fig. 1 Pre-op magnetic resonance imaging (MRI) (A-C) and postoperative MRI of the patient (D-F).
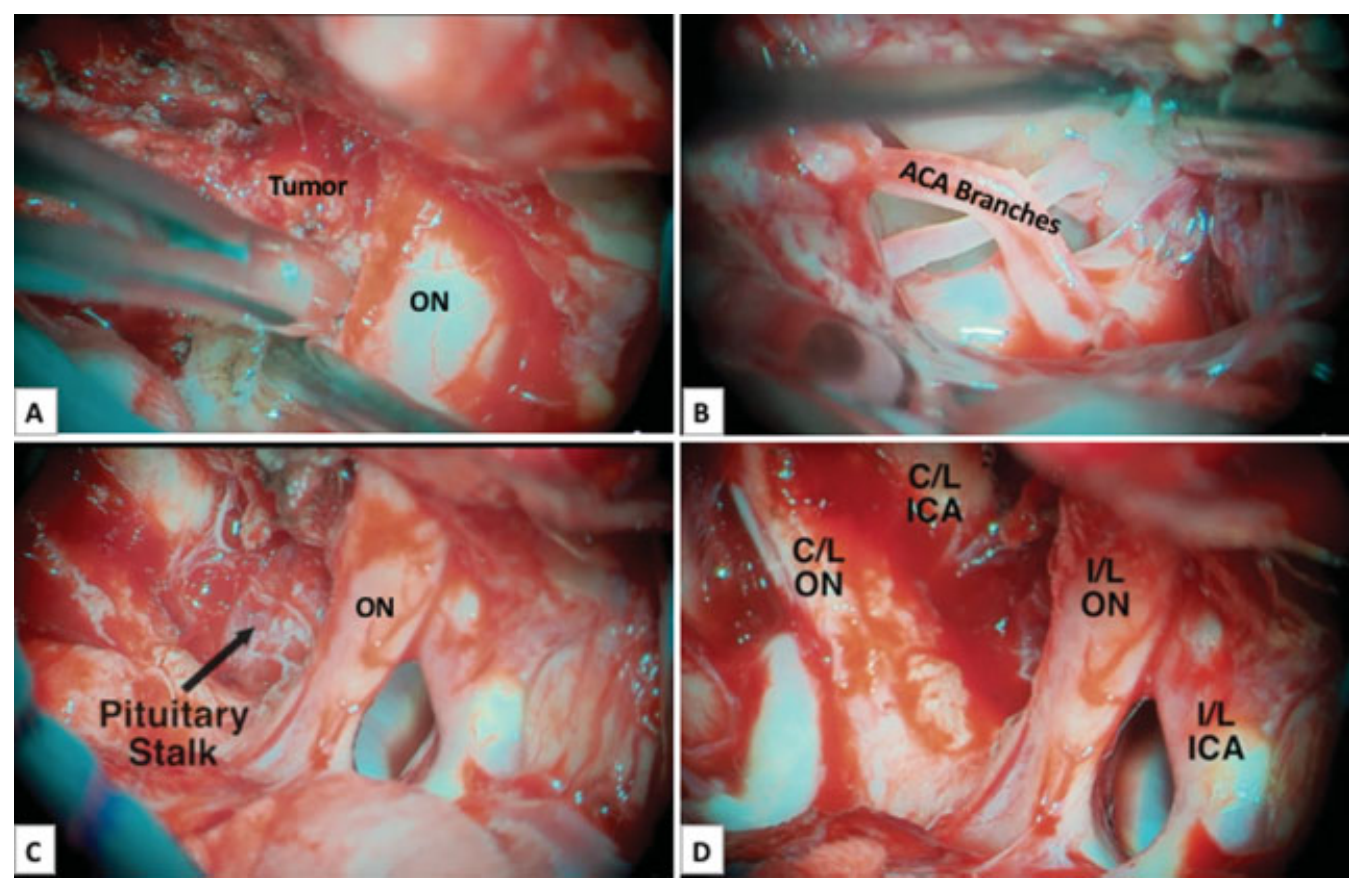

Fig. 2 Intra-op images showing dissection of the tumor from the right optic nerve (A), ACA branches (B), pituitary stalk (C), and the operative view after complete resection of the tumor (D). ON, optic nerve; ACA, anterior cerebral artery; C/L, contralateral; I/L, ipsilateral; ICA, internal carotid artery. 